\title{
A Gestural Account of Neutral Segment Asymmetries in Harmony
}

\author{
Caitlin Smith \\ University of Southern California
}

\section{Introduction}

Harmony is a process by which a feature spreads throughout a word or some other domain. The segment that initiates harmony is referred to as the trigger, and those that undergo harmony are its targets. Some segments seemingly do not participate in harmony; these are called neutral segments, and they are of two types. Transparent segments are those that do not undergo harmony but do not stop it from spreading; they appear to have been skipped by the harmony process. Blockers are those neutral segments that stop the spread of a harmonizing feature.

Transparency in rounding harmony is exemplified in Halh Mongolian (Mongolic; Mongolia, Russia). In Halh, rounding harmony is triggered by a non-high round vowel in an initial syllable (underlined below) and targets following non-high vowels, as in (1a-b). The high front vowel /i/ behaves transparently, as in (1c-d) (Svantesson, Tsendina, Karlsson, \& Franzén 2005). ${ }^{1}$
a. [og-bo] 'give (past)'
c. [po:r-ig-o] 'kidney (acc. refl.)'
b. [ọr-bo] 'enter (past)'
d. [xo:b-ig-o] 'food (acc. refl.)'
cf. [it-be] 'eat (past)'
cf. [pi:r-ig-e] 'brush (acc. refl.)'

Blockers are those neutral segments that stop the spread of a harmonizing feature. This is exemplified by nasal vowel-consonant harmony (hereafter referred to simply as nasal harmony) in Warao (isolate; Venezuela), in which nasality spreads rightward from an underlyingly nasal consonant or vowel (underlined below) and targets vowels and certain consonants, as in (2a-b). However, Warao nasal harmony is blocked by liquids and obstruents, as in (2c-d) (Osborn 1966).
a. [mõj̃õ] 'cormorant'
b. [inã̃̃ããã] 'summer'
c. [nãõte] 'he will come'
d. [nĩhãrapaka] 'alligator'

For some harmony phenomena, such as ATR/RTR harmony, the sets of crosslinguistically attested classes of transparent and blocking segments are identical (see Casali (2008) for a detailed typological survey). However, upon examining the sets of attested neutral segments for rounding harmony and nasal harmony, it is observed that the set of attested transparent segments is a proper subset of attested blocking segments. That is, there are many more classes of segments that are crosslinguistically attested as blockers than as

\footnotetext{
* Many thanks are due to Rachel Walker, Louis Goldstein, Karen Jesney, and Khalil Iskarous for their input on the development of this work. I would also like to thank the members of the Phonlunch group at USC, the phonetics seminar at UCLA, and the audience at AMP 2015 for their helpful comments and questions about this project.

${ }^{1}$ An additional vowel harmony process in Halh divides vowels into pharyngeal and non-pharyngeal classes (Svantesson et al. 2005), which when combined with rounding harmony produces the following alternations:

\begin{tabular}{lcccc} 
& \multicolumn{2}{c}{ non-pharyngeal } & \multicolumn{2}{c}{ pharyngeal } \\
& non-round & round & non-round & round \\
high & $\mathrm{i}$ & $\mathrm{u}$ & & $\mathrm{v}$ \\
non-high & $\mathrm{e}$ & $\mathrm{o}$ & $\mathrm{a}$ & 0
\end{tabular}

(C) 2016 Caitlin Smith

Proceedings of AMP 2015

Completed February 29, 2016
} 
transparent segments. Such patterns of distribution are not predicted by standard approaches to neutrality in harmony, in which neutral segments are the result of a single mechanism within the phonological grammar. In this paper I propose that the adoption of a gesture-based model of harmony accounts for the comparatively limited sets of transparent segments in rounding harmony and nasal harmony, and it does so through two distinct driving forces responsible for transparency and blocking.

The paper is organized as follows. Section 2 outlines the typological asymmetries in the sets of neutral segments in nasal harmony and rounding harmony. Section 3 introduces the Gestural Harmony Model, and Section 4 outlines the workings of two distinct mechanisms of neutrality within the model: transparency via gestural antagonism and blocking via gestural inhibition. Section 5 describes the issue of over-generation of unattested patterns of neutrality in analyses of harmony that use feature co-occurrence constraints. Section 6 concludes.

\section{Typologies of Neutral Segments}

Rounding harmony is common within the Tungusic, Turkic, and Mongolic families. In these languages, rounding harmony is triggered by a round vowel in an initial syllable. Kaun's (1995) survey of rounding harmony systems shows that all vowels are attested blockers in some pattern, with the driving factors being avoidance of cross-height harmony and of non-high round vowels. However, the only attested transparent segments in rounding harmony are the high front vowels $/ \mathrm{i} /$ and $/ \mathrm{I} /$. These patterns and languages exemplifying them are listed in the table below.

Table (1): Attested neutral segments in rounding harmony

\begin{tabular}{lll}
\hline & Neutral Segments & Language \\
\hline \multirow{2}{*}{ Blockers } & $\begin{array}{l}\text { Non-high vowels } \\
\text { High vowels }\end{array}$ & $\begin{array}{l}\text { Turkish (Clements \& Sezer 1982) } \\
\text { Ulcha (Kaun 1995 citing Sunik 1985) }\end{array}$ \\
\hline Transparent segments & High front vowels & Halh Mongolian (Svantesson et al. 2005) \\
\hline
\end{tabular}

A similar asymmetry in the sets of neutral segments is observed in nasal harmony. Nasal harmony is triggered by either a nasal consonant or a nasal vowel, and it may target surrounding consonants and vowels. Looking across all nasal harmony systems, all consonants are attested blockers. Walker (1998/2000) observes that there is a fixed implicational hierarchy of blocking behavior by consonants. Nasal harmony may be blocked by obstruents; obstruents and liquids; or obstruents, liquids, and glides. However, only obstruents are attested as behaving transparently in nasal harmony. This is summarized in the following table.

Table (2): Attested neutral segments in nasal harmony

\begin{tabular}{lll}
\hline & Neutral Segments & Language \\
\hline \multirow{3}{*}{ Blockers } & Obstruents & Kayan (1977) \\
& Obstruents, liquids & Warao (Osborn 1966) \\
& Obstruents, liquids, glides & Sundanese (Robins 1957) \\
\hline Transparent segments & Obstruents & Tuyuca (Barnes \& Takagi de Silzer 1976) \\
\hline
\end{tabular}

To sum up, in both nasal harmony and rounding harmony, blocking and transparency show different patterns of attestation, with transparency limited to a significantly smaller set of segment classes. Attested transparent segment classes make up a proper subset of attested blockers, and an analysis of neutrality in harmony should predict these typological patterns. To that end, I propose that these patterns can be accounted for by treating blocking and transparent segments as the products of two distinct driving forces. This is made possible by adopting a gesture-based representation of harmony: the Gestural Harmony Model, whose workings are outlined in the following section. 


\section{The Gestural Harmony Model}

The Gestural Harmony Model relies on the use of a modified version of gestures as defined within Articulatory Phonology (Browman \& Goldstein 1986, 1989). Gestures are task-based units; they are specified for a goal articulatory state, such as 'open velum,' 'protruded lips,' or 'tongue tip in contact with the alveolar ridge.' They are also specified for the articulators they may recruit to achieve their articulatory tasks, as well as the strength with which they can command these articulators. The achievement of a gesture's articulatory task unfolds over a specified amount of time, the gesture's activation duration. When sufficient time has passed for this task to be achieved, the gesture self-deactivates, and its articulators may return to specified neutral positions until they are recruited by subsequent gestures.

Previous work by (Smith 2015, to appear) has proposed that there is an additional gestural parameter specifying whether a gesture is self-deactivating or not. The following figure compares a typical, selfdeactivating velum opening gesture with a non-self-deactivating velum opening gesture. Both gestures achieve their target articulatory states gradually, represented by the climbing line within each box. Once the target articulatory state is reached, i.e. once the velum is opened, the typical gesture self-deactivates. If the velum is not employed by a subsequent gesture, it is allowed to return to its neutral position (loosely closed). However, the non-self-deactivating gesture remains active beyond the point of target achievement (represented by the dashed line) and the velum remains open.

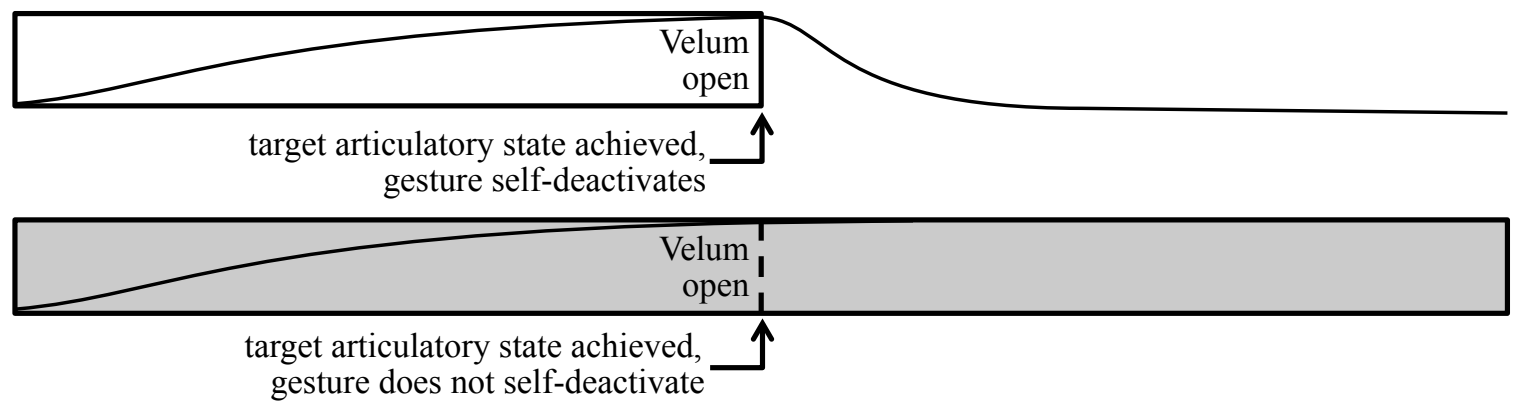

Figure (1): A self-deactivating velum opening gesture and a non-self-deactivating velum opening gesture with extended duration

I propose that in the Gestural Harmony Model harmony is triggered by a gesture that does not selfdeactivate when its target articulatory state is reached. Instead, it remains active, extending in duration and overlapping additional gestures in a word. These overlapped gestures are the targets of harmony. The gesture that is responsible for nasal harmony is a non-self-deactivating velum opening gesture like the one in the figure above. Nasal consonants and vowels that trigger nasal harmony are accompanied by this type of velum opening gesture rather than the typical, self-deactivating velum opening gesture that accompanies nasal segments in non-harmony languages such as English. Similarly, rounding harmony is triggered by a non-self-deactivating lip protrusion gesture that accompanies the lingual gestures of round vowels.

An analysis of rounding harmony within the Gestural Harmony Model is illustrated with an example from Halh Mongolian. Recall from the forms in (1a-b) that rounding harmony in this language is triggered by a non-high round vowel in an initial syllable and targets following non-high vowels. The following figure shows the vocalic portion of a gestural score for the Halh word [og-bo] 'give (past).' The subscripts in the segmental transcription at the top of the figure and in the gestural score itself indicate segment-togesture correspondence. The vowel /o/ is made up of a lingual gesture specified for a constriction in the uvular-pharyngeal region of the vocal tract as well as a lip protrusion gesture responsible for rounding. The first /o/ in [og-3o] is rendered a harmony trigger by virtue of the fact that its lip protrusion gesture is nonself-deactivating. This lip protrusion gesture extends to overlap the lingual gesture of the second vowel as well, and so it surfaces as rounded. This is harmony in the Gestural Harmony Model. 


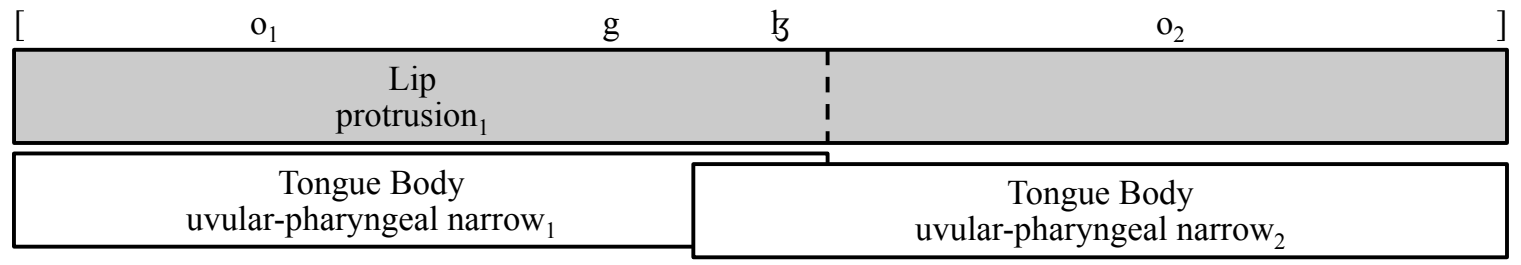

Figure (2): Gestural score for the Halh word [og-bo] 'give (past)' with non-self-deactivating lip protrusion gesture

A similar analysis can be provided for nasal harmony as well. Recall from the data in (2a-b) that in Warao nasality spreads rightward from a nasal segment and targets vowels and glides. The following figure shows a gestural score for the fully harmonizing Warao word [mõjõ] 'cormorant,' in which the word-initial $[\mathrm{m}]$ triggers nasal harmony. A nasal consonant is always made up of a consonantal constriction gesture (in the case of [m], the labial closure gesture) as well as a velum opening gesture. Here, the velum opening gesture accompanying the word-initial $[\mathrm{m}]$ is non-self-deactivating, causing it to remain active throughout the entire word. The following gestures are overlapped by the velum opening gesture, and so they surface as nasalized.

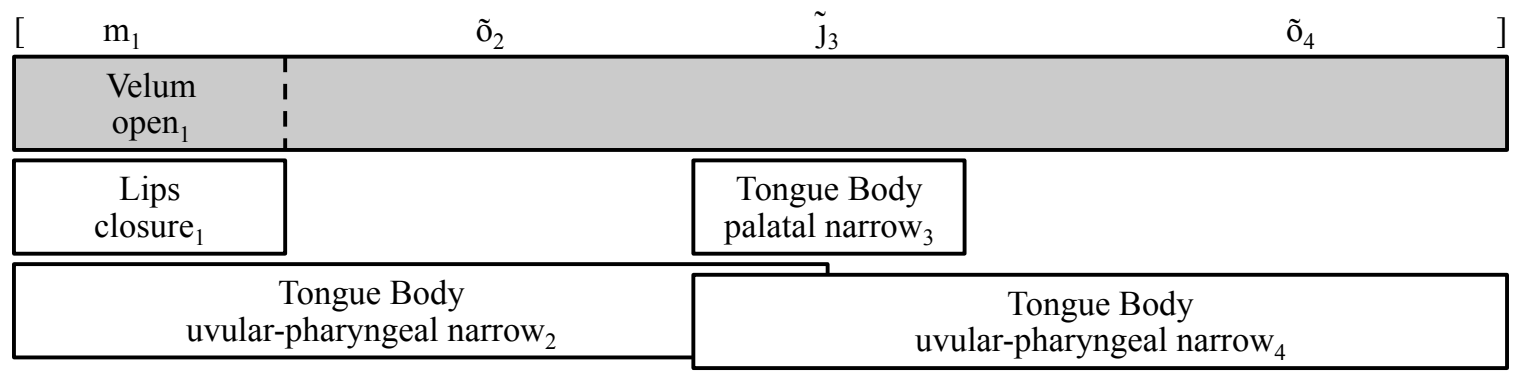

Figure (3): Gestural score for the Warao word [mõjõ] 'cormorant' with non-self-deactivating velum opening gesture

The forms illustrating Halh Mongolian rounding harmony and Warao nasal harmony examined thus far have featured a harmonizing gesture that overlaps all other gestures in a word without issue, causing them to undergo harmony. The following section examines how to represent the non-undergoing behavior of neutral segments in harmony.

\section{Neutrality in the Gestural Harmony Model}

The Gestural Harmony Model provides for an analysis of transparency and blocking as the results of distinct mechanisms of neutrality within harmony. Crucially, the mechanism responsible for transparency in the Gestural Harmony Model is available to a limited set of segments, accounting for the fact that transparent segments make up a proper subset of neutral segments in nasal harmony and rounding harmony. The blocking mechanism, on the other hand, is available to all neutral segments. This section introduces these two mechanisms in turn and describes their distinct but related phonetic motivations.

4.1 Incompatibility \& Antagonism The preceding section proposed that harmony is the result of a gesture that potentially overlaps all other gestures in a word, causing them to undergo harmony. However, at times this sort of gestural overlap may result in the concurrent activation of gestures that are (1) incompatible or (2) antagonistic. These two possible consequences of gestural overlap are the motivators of the two distinct types of neutrality within the Gestural Harmony Model.

Gestural incompatibility refers to any situation in which concurrent activation of two gestures is marked in some way. The overlapping of their goal articulatory states results in the production of a segment that is either articulatorily or perceptually difficult. For example, the concurrent activation of a velum opening gesture with the lingual gestures making up a liquid is marked for perceptual reasons (see Ohala 
(1975) for discussion). Therefore, these sets of gestures are considered incompatible, or marked. A language's phonological grammar may either allow the marked structure caused by this gestural overlap, or ban it and prevent the overlap of these incompatible gestures.

Two gestures are antagonistic if they have directly opposing articulatory goal states that pull an articulator in opposite directions. For example, a velum opening and a velum closure gesture are antagonistic when active at the same time. During this period of concurrent activation, the velum receives conflicting instructions from the two active gestures; these goal articulatory states cannot both be achieved. Instead, resolution of this competition is calculated according to the Task Dynamic Model of speech production (Saltzman \& Munhall 1989). According to the Task Dynamic Model, two gestures with antagonistic target articulatory states undergo gestural blending, or averaging. During the period of their concurrent activation, the blended goal articulatory state of two gestures is the weighted average of their individual goal states, with each gesture's specified strength parameter contributing the weights. As a result of this blending, one or both of the antagonistic gestures will not fully achieve its target articulatory state. It should be noted that gestural antagonism is a form of gestural incompatibility; resolving the conflict between two antagonistic goal states via gestural blending is articulatory marked. However, it is not the case that incompatibility between gestures entails gestural antagonism; gestures may be incompatible for a variety of reasons not necessarily having to do with gestural antagonism.

The distinct treatments of transparency and blocking in the Gestural Harmony Model come down to these two different consequences of potential gestural overlap and whether a phonological grammar tolerates such overlap. Transparency is the result of the concurrent activation of two antagonistic gestures. Blocking, on the other hand, is the result of a ban on the concurrent activation of two incompatible gestures. The following subsections address each of these proposals in turn.

4.2 Coactivation Transparency Transparency results when a harmonizing gesture and one of the gestures it overlaps are antagonistic to one another. When this occurs, gestural blending favors the highstrength gesture of the transparent segment, and it appears not to have undergone harmony. Gestural antagonism arises in very few instances of gestural overlap, which explains why very few segment classes may behave transparently in harmony. This is demonstrated first by an examination of transparency to rounding harmony in Halh Mongolian.

Recall that in Halh the high front vowel /i/ is transparent to rounding harmony, as in (3) (repeated from $(1 \mathrm{c}-\mathrm{d}))$ :

a. [po:r-ig-o] 'kidney (acc. refl.)'

b. [xo:b-ig-o] 'food (acc. refl.)'

cf. [pi:r-ig-e] 'brush (acc. refl.)'

I claim that high front vowels are transparent to nasal harmony in Halh and other languages due to their gestural makeup. A high front vowel is represented by both a palatal constriction gesture and, most importantly for the purpose of representing transparency, a lip spreading gesture. The active control of lip spreading has been posited as a means of raising the second formant of high front vowels in order to maximize their perceptual distance from back vowels. The presence of this active lip spreading is supported by several articulatory studies, including Hadding, Hirose, \& Harris (1976), Sussman \& Westbury (1981), and Goldstein (1991).

Figure (4) depicts the vocalic portion of the gesture score for the Halh Mongolian word [po:r-ig-o] 'kidney (acc. refl.).' As in figure (2), a lip protrusion gesture extends throughout the word, resulting in rounding harmony. However, here its overlap with the gestures of /i/ results not in rounding of the vowel, but in transparency to rounding harmony. The lip spreading gesture included in the representation of $/ \mathrm{i} /$ is antagonistic to the lip protrusion gesture that overlaps it. Because the lip spreading gesture is specified for a high gestural strength (denoted by ' $\mathrm{S}$ ') while the harmonizing lip protrusion gesture is specified for a low gestural strength (denoted by ' $\mathrm{W}$ '), blending of their antagonistic articulatory goals is resolved in favor of the lip spreading gesture of $/ \mathrm{i} /$. As a result, the lips are pulled away from their protruded position and instead spread during the production of $/ \mathrm{i} /$. When the production of /i/ ceases, the lips are free to return to the protruded position required by the lip protrusion gesture. 


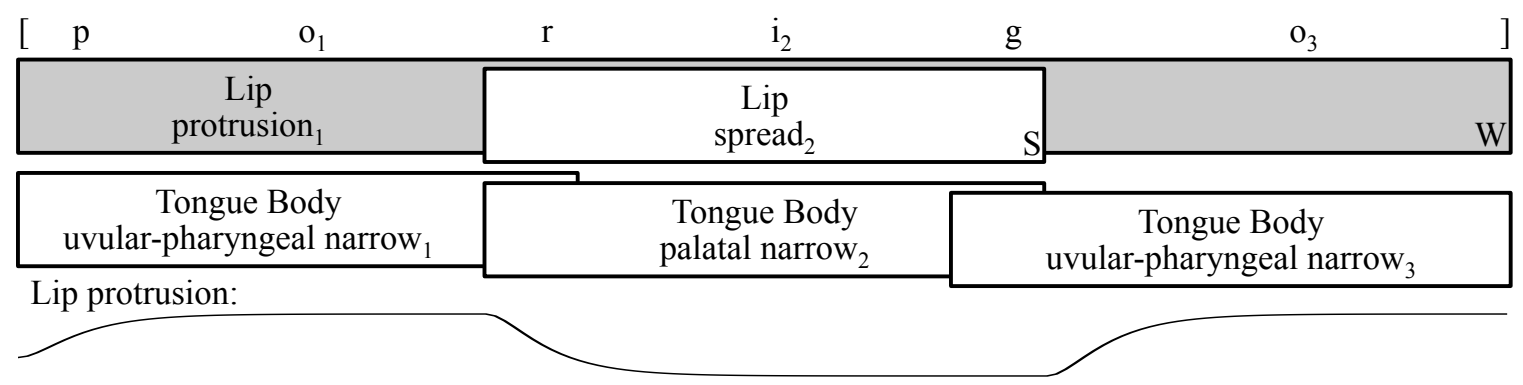

Figure (4):Gestural score for the Halh word [po:r-ig-o] 'kidney (acc. refl.)' with non-self-deactivating lip protrusion gesture and antagonistic lip spreading gesture

Note that despite the brief lack of rounding during the vowel [i], the harmonizing lip protrusion gesture is active throughout the entire word. It is simply prevented from achieving its goal articulatory state during the production of the high front vowel /i/. This temporary masking of the effect of a harmonizing gesture by a concurrently active antagonistic gesture is referred to as coactivation transparency.

The same explanation based on gestural antagonism can be extended to obstruent transparency in nasal harmony. In Tuyuca (Tucanoan; Colombia) root morphemes are either nasal or oral, indicating that the activation duration of any velum opening gesture present in a root will span the entirety of that root. The forms in (4a-c) exemplify these all-nasal roots. However, obstruents do not undergo this nasal harmony, but are instead transparent to it, as in (4d-f) (Barnes \& Takagi de Silzer 1976; Barnes 1996).
a. [jãmĩ] 'night'

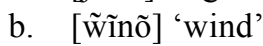
d. [mĩpĩ] 'badger'

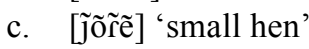
e. [w̃ãtĩ] 'demon'
f. [j̃̃̃sõ] 'bird'

As in the case of Halh rounding harmony, obstruent transparency in nasal harmony is the result of the gestural representation of obstruents. Obstruents are multi-gestural segments; they include an oral constriction gesture, possibly some kind of glottal gesture, and a velum closure gesture. This velum closure gesture is posited for its ability to raise the velum from its neutral, loosely closed position in order to ensure that there is an especially tight seal of the velopharyngeal port. This tight seal prevents nasal leakage and creates the aerodynamic conditions necessary for obstruency. The inclusion of a velum closure gesture for obstruents is supported by experimental results that show raising of the velum during the production of oral stops, even when surrounded by oral vowels (Lubker 1968; Bell-Berti \& Hirose 1975; Bell-Berti 1976).

The velum closure gesture responsible for obstruency is antagonistic to the velum opening gesture responsible for nasal harmony, resulting in the transparent behavior of obstruents in nasal harmony. The following figure demonstrates with a gestural score for the Tuyuca word [mĩpĩ] 'badger.' A non-selfdeactivating velum opening gesture overlaps all other gestures in the word. The initial consonant and the vowels surface as nasalized, but the obstruent $[\mathrm{p}]$, also overlapped by the velum opening gesture, surfaces as oral. This is due to the presence of a velum closure gesture that is antagonistic to the harmonizing velum opening gesture. The velum closure gesture is specified for a high gestural strength, allowing it to counteract the effect of the velum opening gesture during the period of their concurrent activation. Once the production of the obstruent has concluded and the velum closure gesture deactivates, there is no longer an active gesture that is antagonistic to the velum opening gesture, and the velum opens once again. 


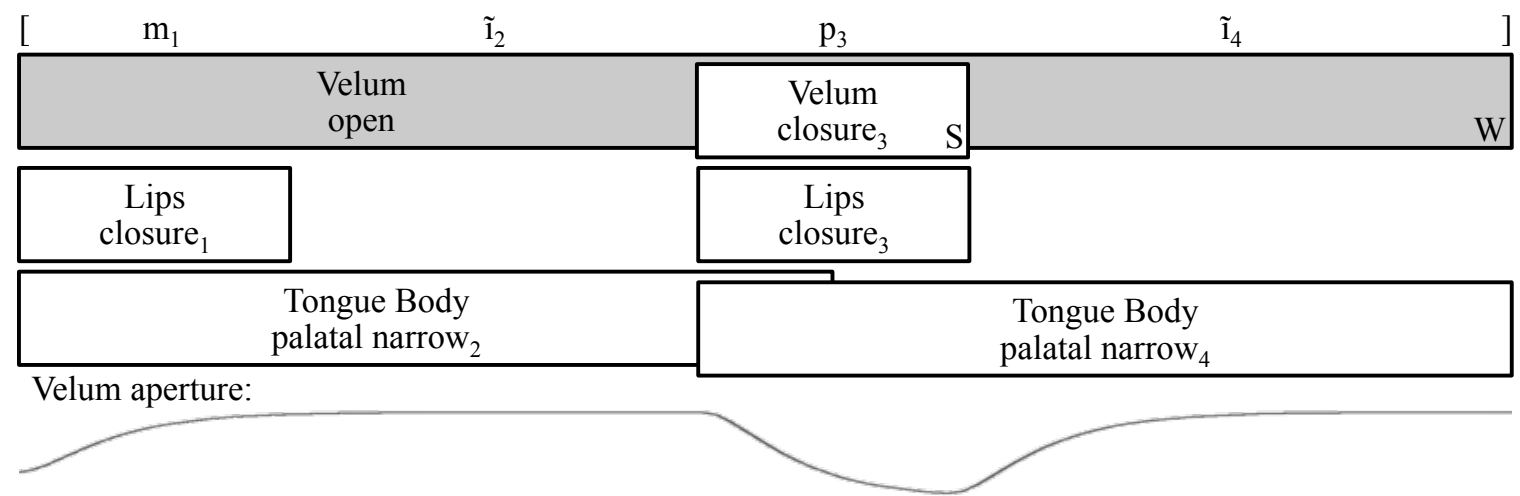

Figure (5): Gestural score for the Tuyuca word [mĩpĩ] 'badger' with non-self-deactivating velum opening gesture and antagonistic velum closure gesture

The analysis of transparency in the Gestural Harmony Model takes advantage of the goal-based, spatiotemporal nature of gestures to account for the fact that the sets of possible transparent segments in rounding harmony and nasal harmony are restricted and specific to the involved articulators. Transparency arises when a harmonizing gesture is temporarily prevented from achieving its goal articulatory state by the concurrent activation of a stronger gesture with a directly opposing goal state. Therefore, the coactivation transparency account predicts that only those segments bearing gestures that are antagonistic to a harmonizing gesture can be transparent. This means that only velum closure-bearing obstruents can be transparent to nasal harmony, and only lip spreading-bearing high front vowels can be transparent to rounding harmony.

4.3 Blocking via Gestural Inhibition In the Gestural Harmony Model, blocking of harmony is not the result of concurrent activation of two gestures, as was shown to be the case for transparency. Rather, it is the result of a ban on the temporal overlap of a harmonizing gesture and a blocking gesture in order to avoid the incompatibility of their coproduction. Recall that incompatible gestures are those whose coproduction is articulatorily or perceptually marked. Because there are many sources of gestural incompatibility, both articulatory and perceptual, blockers are predicted to be prevalent within harmony systems.

If the concurrent production of a harmonizing gesture and an incompatible blocking gesture is to be avoided, the two gestures must be prevented from being active at the same time. Therefore, the harmonizing gesture must deactivate when the blocking gesture activates. However, a harmonizing gesture is specified as being unable to deactivate itself; it is precisely this aspect of the gesture that renders it a trigger of harmony. Instead, it is the activation of the blocking gesture that causes the deactivation of the non-self-deactivating gesture. I propose that this is achieved through a new type of inter-gestural relation: an inhibition relation on gestural activation. This relation is part of the representation of a phonological form, and can be formally represented as in the following figure.

\section{Inhibited Gesture $\mathbf{X}$. . . . Inhibitory Gesture}

Figure (6): An asymmetric inhibition relation between gestures

The inhibition relation that is posited to exist between harmonizing and blocking gestures is asymmetric, with the blocking gesture acting as an inhibitor of the harmonizing gesture. When an inhibitory gesture is activated, it leeches the activation from the inhibited gesture, as shown in the timecourse in the following figure. The dashed line represents the activation level of the inhibited gesture, which deactivates upon the activation of the inhibitory gesture, represented by the solid line. 


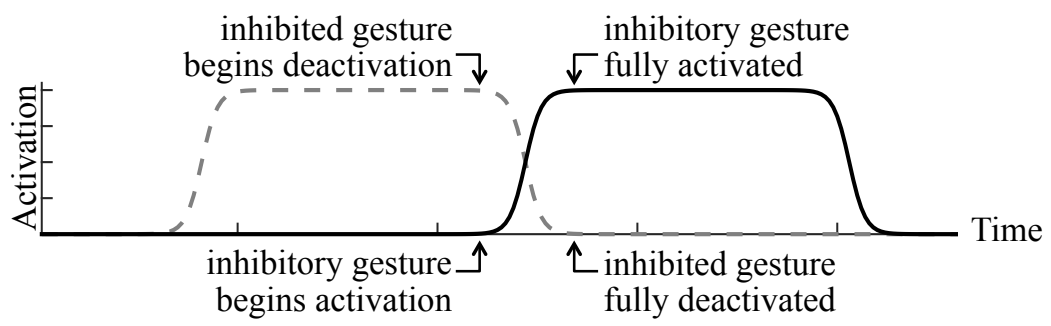

Figure (7): Timecourse of gestural activation for two gestures in an inhibition relation

A phonological grammar can require an inhibition relation between any two sets of incompatible gestures in order to prevent their concurrent activation. Blocking via gestural inhibition is illustrated here with an example from nasal harmony in Warao. Recall that some of the Warao forms provided earlier showed blocking of nasal harmony by liquids and obstruents. This blocking behavior can now be accounted for via an inhibition relation between the blocking gestures of liquids and obstruents and the non-selfdeactivating velum opening gesture responsible for nasal harmony. The following figure depicts a gestural score for the Warao word [nĩhãapaka] 'alligator'' in which the liquid [r] blocks nasal harmony. (For reasons of space, only the first three syllables are included in the gestural score.) Since a restriction against the overlap of a velum opening gesture and the gesture(s) of a liquid is active within the phonological grammar of Warao, overlap is prevented via the inclusion of an inhibition relation between the gesture of the liquid $[\mathrm{r}]$ and the harmonizing velum opening gesture. Upon activation of the gesture of $[\mathrm{r}]$, the harmonizing velum opening gesture of the triggering $[\mathrm{n}]$ deactivates.

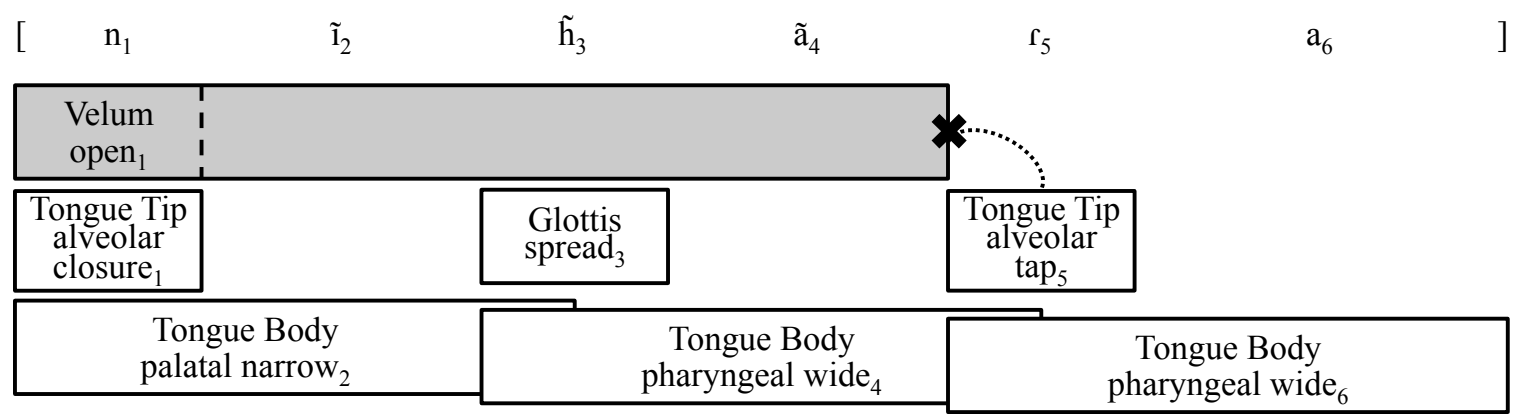

Figure (8): Gestural score for the first three syllables of the Warao word [nĩ̄ãapaka] 'alligator' with nonself-deactivating velum opening gesture deactivated via inhibition relation

It should be noted that segments that behave transparently in one language may act as blockers of harmony in another. While in Warao obstruents behave as blockers, the previous section provided examples of obstruents behaving transparently in Tuyuca. The following figure depicts a gestural score for the Warao form [nãõte] 'he will come,' in which the obstruent [t] blocks nasal harmony. This comes down to a difference in whether a language allows overlap between a non-self-deactivating velum opening gesture and the gestures of an obstruent, resulting in transparency, or whether it bans that overlap due to gestural incompatibility, resulting in blocking. 


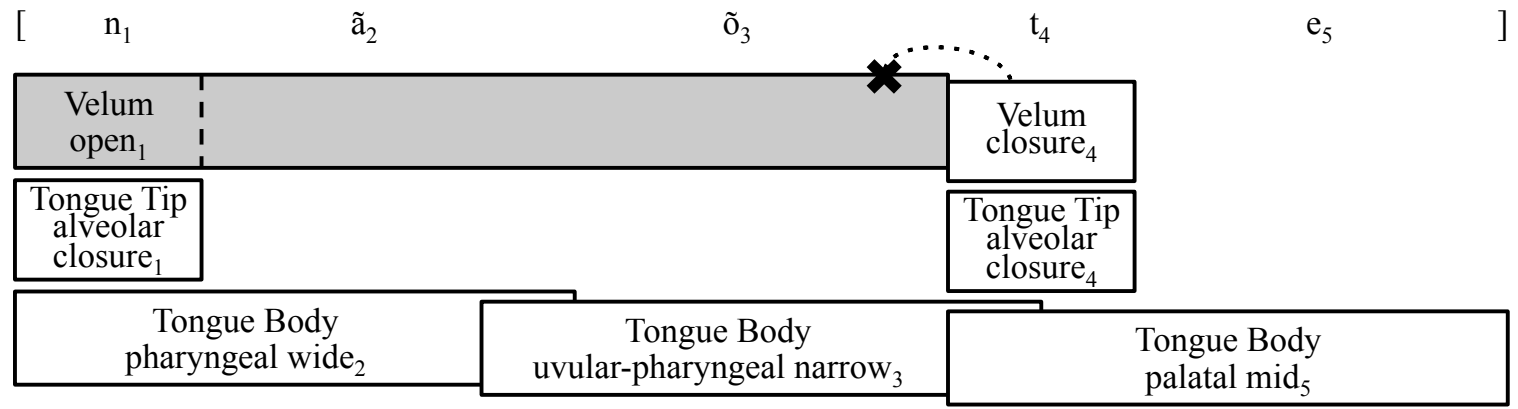

Figure (9): Gestural score for the Warao word [nãote] 'he will come' with non-self-deactivating velum opening gesture deactivated via inhibition relation

While an obstruent such as /t/ may behave either transparently or as a blocker depending upon an individual language's phonological grammar, a liquid such as / $\mathrm{f} /$ in the Warao form [nĩ̄̃ãrapaka] 'alligator' may never behave transparently. If a language such as Warao restricts the overlap of a non-selfdeactivating velum opening gesture and the gesture of a liquid such as $/ \mathrm{f} /$, then $/ \mathrm{f} /$ will block nasal harmony. If such a restriction is not active in the grammar, $/ \mathrm{f} /$ will be overlapped by the velum opening gesture and will undergo harmony. Such is the case in the Tuyuca form [jõõẽ] 'small hen,' in which / $/$ does not block nasal harmony but instead undergoes it, as demonstrated in the following figure. Because $/ \mathrm{r} /$ is not accompanied by an antagonistic velum closure gesture, it cannot surface as transparent to nasal harmony. Thus, the Gestural Harmony Model succeeds in restricting the mechanism responsible for transparency to a small set of segments while allowing a larger set of segments to behave as blockers of harmony.

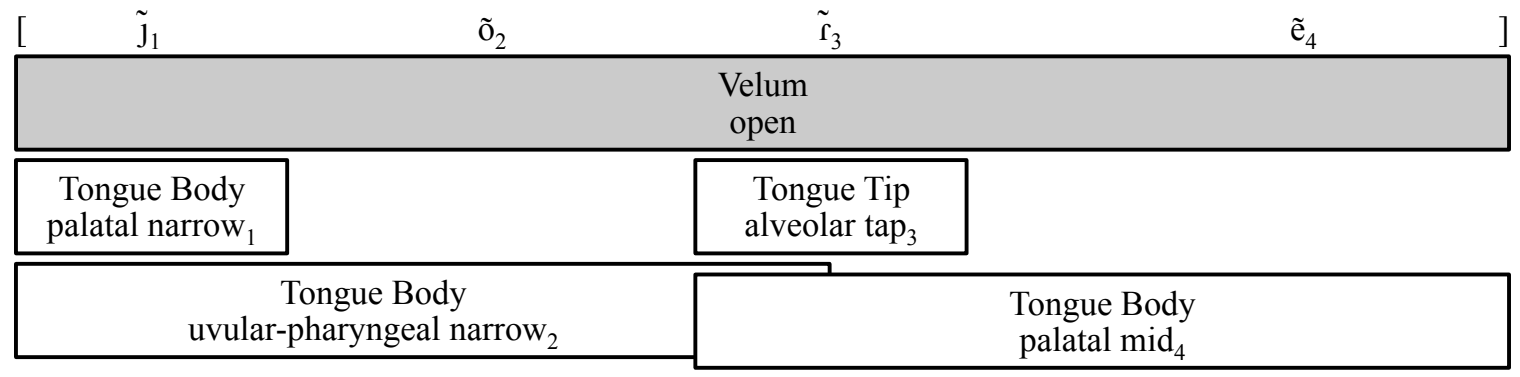

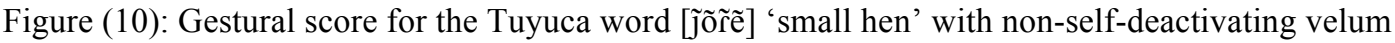
opening gesture overlapping all other gestures

Gestural inhibition can also account for patterns of blocking in rounding harmony, as illustrated here with an example from rounding harmony in Ulcha (Tungusic; Russia). In Ulcha non-high round vowels trigger rounding harmony and are targeted by it $(7 a-b)$, while high vowels, including the high round vowels $/ \mathrm{u} /$ and $/ \mathrm{v} /$, act as blockers (7c-d) (Kaun 1995). ${ }^{2}$
a. [goro] 'far'
c. [do:krla] 'inside' *[do:kilo]
b. [totongo] 'multi-colored'
d. [korvka] 'pike (fish) skin' $*[\mathrm{kor} r \mathrm{ko}]$

This blocking can be analyzed as the result of an inhibition relation between the gesture(s) of a high vowel and a non-self-deactivating lip protrusion gesture. The following figure depicts a gestural score for the word [do:kıla] 'inside,' in which the gestures of the high vowel / $\mathrm{I} /$ inhibit the non-self-deactivating lip protrusion gesture of $/ \mathrm{o} /$. This results in the deactivation of the lip protrusion gesture and the prevention of its overlap with the gestures of $/ \mathrm{I} /$.

\footnotetext{
${ }^{2}$ According to Kaun, Ulcha also displays RTR harmony, leading to the alternations $/ \mathrm{u} / \sim / \mathrm{\mho} /, / \mathrm{i} / \sim / \mathrm{I} /$, and $/ \mathrm{a} / \sim / \mathrm{\jmath} /$.
} 


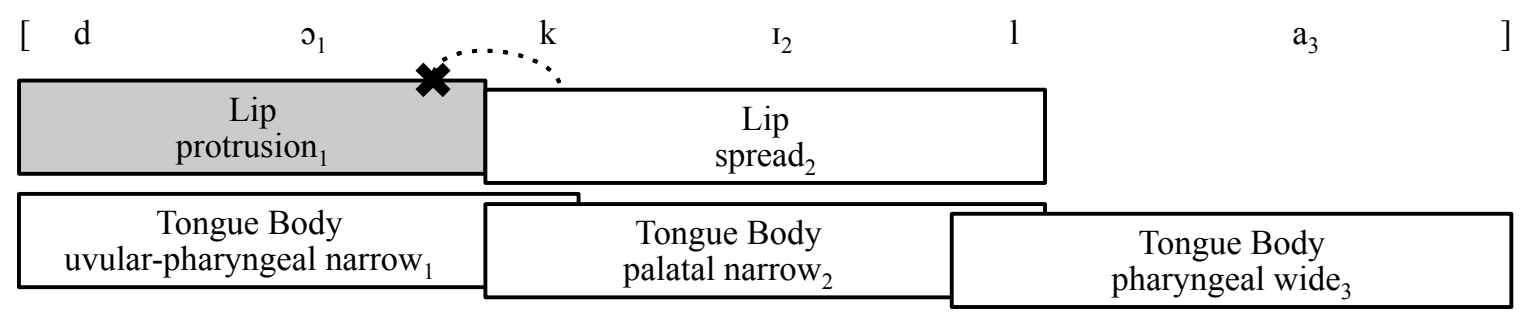

Figure (11): Gestural score for the Ulcha word [do:kıla] 'inside' with non-self-deactivating lip protrusion gesture deactivated via inhibition relation

Of note here is the fact that in Ulcha /i/ and / $/$ / behave as a blocker of rounding harmony, whereas in Halh Mongolian /i/ is transparent to rounding harmony, as seen in the previous subsection. The difference between these two patterns lies in whether or not the restriction on the overlap of a high vowel such as /i/ and a lip protrusion gesture is active within a language's phonological grammar. In Ulcha, the constraint responsible for preventing the overlap of these gestures is presumably high-ranked, necessitating the inclusion of an inhibition relation in high vowel-containing forms such as [do:kıla]. In contrast, this restriction is not active in the grammar of Halh Mongolian, and overlap between the lip protrusion gesture and the gestures of $/ \mathrm{i} /$ is permitted. As demonstrated in the previous subsection, this overlap results in transparency of high front vowels rather than their audibly undergoing rounding harmony. ${ }^{3}$

A ban on gestural overlap that may be active within a phonological grammar is essentially the gestural equivalent of a co-occurrence constraint. In this way, the gestural analysis of blocking in harmony does not differ much from any featural analysis of blocking based on co-occurrence constraints. The only difference is that in the Gestural Harmony Model these bans on the co-occurrence of incompatible gestures are used only in the analysis of blocking, and not for both blocking and transparency. The following section addresses the issue of over-generation of predicted transparent segments in featural analyses that utilize cooccurrence constraints to account for the patterning of all neutral segments.

\section{Alternative Accounts of Neutrality}

While the Gestural Harmony Model utilizes two distinct mechanisms to account for transparency and blocking, in many featural accounts of harmony all neutral segments are accounted for via the same mechanism within the phonological grammar. There are numerous analyses of harmony that utilize feature co-occurrence constraints to account for the harmony-neutral status of both blockers and transparent segments. These include embedded feature domains (Smolensky 1993), grounded path conditions (Archangeli \& Pulleyblank 1994), Optimal Domains Theory (Cole \& Kisseberth 1994, 1995), targeted constraints (Bakovic \& Wilson 2000; Wilson 2003), Sympathy Theory (Walker 1998/2000), and Span Theory (McCarthy 2004; O'Keefe 2005). Though all of these approaches differ in the details of their implementation, they all make the undesirable prediction that all attested blockers within a harmony phenomenon should be able to behave transparently as well, contrary to the crosslinguistic facts. This is demonstrated with an example from Optimal Domains Theory (ODT), though this issue is not specific to that framework.

Analyses within ODT rely upon three main constraint types. The harmony driver, WIDESCOPEALIGNMENT (WSA), requires a feature domain to extend as much as necessary in order to be aligned with the edge of some domain. The constraint EXPRESSION requires every segment within the extended feature domain to bear that feature; it is violated by transparent segments. Finally, a cooccurrence constraint is utilized to penalize harmony when it creates a marked segment; this constraint drives neutrality. In ODT, neutrality is the result of a high-ranked co-occurrence constraint that prevents a segment from taking on a harmonizing feature. Whether that neutrality takes the form of transparency or

\footnotetext{
${ }^{3}$ In both Halh Mongolian and Ulcha, high back round vowels $/ \mathrm{u} /$ and $/ \mathrm{v} /$ do not trigger rounding harmony and also act as blockers. While a full treatment is not included here, this pattern can be attributed to a ban on the overlap between a high back vowel gesture and a non-self-deactivating lip protrusion gesture. Because of this ban, a high back round vowel will block rounding harmony and will surface only with a typical, self-deactivating lip protrusion gesture.
} 
blocking is the result of the ranking of the other two constraints. When co-occurrence and WSA are ranked above EXPRESSION, the result is transparency. When co-occurrence and EXPRESSION are ranked above WSA, the result is blocking.

Full harmony: WIDESCOPEALIGNMENT, EXPRESSION $>>$ Co-occurrence Transparency: Co-occurrence, WIDESCOPEALIGNMENT $>>$ EXPRESSION Blocking: Co-occurrence, EXPRESSION $\gg$ WIDESCOPEALIGNMENT

However, this analysis makes an unwanted prediction: any attested blocking segment that is the subject of a co-occurrence constraint may also behave transparently, and this transparency in easily achieved by simple constraint reranking. This is illustrated with an example from nasal harmony. Liquids are attested blockers of nasal harmony; therefore, Walker (1998/2000) proposes the co-occurrence constraint *NASALLIQUID. Blocking of nasal harmony by liquids is achieved in ODT via the ranking *NASALLIQUID, EXPRESSION $>$ WSA(nasal). However, by reranking these constraints so that WSA(nasal) and *NASALLIQUID outrank EXPRESSION, the grammar generates a language in which liquids are transparent to nasal harmony, which is an unattested pattern. The same problem arises for neutral segments in rounding harmony. Kaun (1995) proposes the constraint * ROLO (no non-high round vowels) to account for the blocking behavior exhibited by non-high vowels in some rounding harmony systems. With the ranking of WSA(round) and *ROLO over EXPRESSION the grammar generates a language in which non-high vowels are transparent to rounding harmony, which is unattested.

ODT and similar frameworks in which both blocking and transparent segments are treated as the result of the same constraints in different rankings will over-generate possible patterns of transparency. The Gestural Harmony Model avoids this by only using co-occurrence restrictions (in the form of restrictions on incompatible gestural overlap) in its analysis of blocking, and not of transparency. Instead, transparency is restricted only to those segments that include a gesture that is antagonistic to the harmonizing gesture, correctly constraining predictions as to which segments should behave transparently within a given harmony phenomenon.

\section{Conclusion}

This paper has introduced the Gestural Harmony Model and its two distinct mechanisms of neutrality in harmony as a means of accounting for the neutral segment asymmetries in nasal harmony and rounding harmony. Coactivation transparency is accounted for via concurrent activation of antagonistic gestures, while blocking is the result of a ban on temporal overlap of incompatible gestures, captured by an inhibition relation between them. This approach avoids over-generation of predicted transparent segments in harmony systems that display asymmetries in the attested sets of neutral segments, specifically nasal harmony and rounding harmony. This makes a strong case for the gestural representation of harmony processes, as it provides a better match to the typological facts.

This paper has focused on rounding harmony and nasal harmony as cases in which crosslinguistically attested sets of transparent segments make up a proper subset of attested neutral segments. However, other harmony phenomena, such as ATR/RTR harmony, height harmony, and backness harmony do not display such asymmetries in attested neutral segments. While such a pattern is not inconsistent with the workings of the Gestural Harmony Model, further work is necessary to determine to what extent the presence or absence of a neutral segment asymmetry within a given harmony phenomenon is a product of the involved articulators.

\section{References}

Archangeli, Diana, \& Pulleyblank, Douglas. (1994). Grounded Phonology. MIT Press.

Bakovic, Eric, \& Wilson, Colin. (2000). Transparency, Strict Locality, and Targeted Constraints. In Billerey \& Lillehaugen (Eds.), Proceedings of the Nineteenth West Coast Conference on Formal Linguistics (WCCFL 19) (pp. 43-56). Somerville, MA: Cascadilla Press.

Barnes, Janet. (1996). Autosegments with Three-Way Lexical Contrasts in Tuyuca. International Journal of American Linguistics, 62(1), 31-58. 
Barnes, Janet, \& Takagi de Silzer, Sheryl. (1976). Fonología del tuyuca. In Sistemas fonológicos de idiomas colombianos, Tomo III (pp. 123-137). Lomalinda: Editorial Townsend.

Bell-Berti, Fredericka. (1976). An Electromyographic Study of Velopharyngeal Function in Speech. Journal of Speech and Hearing Research, 19(2), 225-240.

Bell-Berti, Fredericka, \& Hirose, Hajime. (1975). Palatal activity in voicing distinctions: a simultaneous fiberoptic and electromyographic study. Journal of Phonetics, 3, 69-74.

Blust, Robert A. (1977). Sketches of the Morphology and Phonology of Bornean Languages 1: Uma Juman (Kayan). Papers in Borneo and Western Linguistics No. 2. Pacific Linguistics, 7-122.

Browman, Catherine P., \& Goldstein, Louis. (1986). Towards an Articulatory Phonology. Phonology Yearbook, 3, 219-252.

Browman, Catherine P., \& Goldstein, Louis. (1989). Articulatory gestures as phonological units. Phonology, 6(2), 201251.

Casali, Roderic F. (2008). ATR Harmony in African Languages. Language and Linguistics Compass, 2(3), 496-549.

Clements, G. N., \& Sezer, Engin. (1982). Vowel and consonant disharmony in Turkish. In H. van der Hulst \& N. Smith (Eds.), The Structure of Phonological Representations (Part II) (pp. 213-255). Dordrecht: Foris Publications.

Cole, Jennifer, \& Kisseberth, Charles. (1994). An Optimal Domains Theory of Harmony. Studies in the Linguistic Sciences, 24(2), 101-114.

Cole, Jennifer, \& Kisseberth, Charles. (1995). Nasal Harmony in Optimal Domains Theory. In Proceedings of the Western Conference on Linguistics (pp. 44-58).

Goldstein, Louis. (1991). Lip Rounding as Side Contact. In Proceedings of the 12th International Congress of Phonetic Sciences (pp. 97-101). Aix-en-Provence: Université de Provence.

Hadding, Kerstin, Hirose, Hajime, \& Harris, Katherine S. (1976). Facial muscle activity in the production of Swedish vowels: an electromyographic study. Journal of Phonetics, 4, 233-245.

Kaun, Abigail. (1995). The Typology of Rounding Harmony: An Optimality Theoretic Approach. Ph.D. Dissertation, University of California Los Angeles.

Lubker, James F. (1968). An Electromyographic-Cinefluorographic Investigation of Velar Function During Normal Speech Production. Cleft Palate Journal, 5, 1-18.

McCarthy, John J. (2004). Headed spans and autosegmental spreading. Unpublished Manuscript, University of Massachusetts Amherst.

O'Keefe, Michael. (2005). Transparency in Span Theory. In University of Massachusetts Occasional Papers in Linguistics 33: Papers in Optimality Theory 3. Amherst, MA.

Ohala, John J. (1975). Phonetic explanations for nasal sound patterns. In C. A. Ferguson, L. M. Hyman, \& J. J. Ohala (Eds.), Nasálfest: Papers from a Symposium on Nasals and Nasalization (pp. 289-316). Stanford, CA.

Osborn, Henry A. (1966). Warao I: Phonology and Morphophonemics. International Journal of American Linguistics, 32(2), 108-123.

Robins, R. H. (1957). Vowel Nasality in Sundanese: A Phonological and Grammatical Study. In Studies in Linguistic Analysis (pp. 87-103). London: Basil Blackwell.

Saltzman, Elliot, \& Munhall, Kevin G. (1989). A Dynamical Approach to Gestural Patterning in Speech Production. Ecological Psychology, 1(4), 333-382.

Smith, Caitlin. (2015). Nasal Harmony as Non-Automatic Gestural Deactivation. Unpublished Manuscript, University of Southern California.

Smith, Caitlin. (to appear). Nasal Spreading as Defective Gestural Deactivation. In Supplemental Proceedings of the 2014 Annual Meeting on Phonology.

Smolensky, Paul. (1993). Harmony, Markedness, and Phonological Activity. In Rutgers Optimality Workshop I (ROWI).

Sussman, Harvey M., \& Westbury, John R. (1981). The Effects of Antagonistic Gestures on Temporal and Amplitude Parameters of Anticipatory Labial Coarticulation. Journal of Speech and Hearing Research, 46, 16-24.

Svantesson, Jan-Olof, Tsendina, Anna, Karlsson, Anastasia, \& Franzén, Vivan. (2005). The Phonology of Mongolian. Oxford University Press.

Walker, Rachel. (1998). Nasalization, Neutral Segments, and Opacity Effects. Ph.D. Dissertation, University of California Santa Cruz.

Wilson, Colin. (2003). Analyzing unbounded spreading with constraints: marks, targets, and derivations. Unpublished Manuscript, University of California Los Angeles. 